\title{
De la economía tradicional a la economía digital compartida
}

\section{From traditional economy to shared digital economy}

Jaime Enrique Sarmiento Suárez

José Luis Garcés Bautista

Universidad Pontifica Bolivariana, Colombia

Autor para correspondencia: jaime.sarmientos@upb.edu.com, jose.garces@upb.edu.com

Fecha de recepción: 24 de Agosto de 2017 - Fecha de aceptación: 30 de Septiembre de 2017

Resumen: La economía mundial viene sufriendo unos grandes cambios, los cuales han llevado a que la economía tradicional basada en un hiperconsumismo se vea amenazada y desplazada por una nueva tendencia económica basada en un consumo inteligente y sostenible, un consumo que se preocupa por no utilizar más de lo que sea necesario, un consumo basado en la colaboración y en el empleo de aquellos bienes ociosos. Este nuevo fenómeno se deriva de la crisis económica que estaba afrontando el mundo en la última década, el apogeo de las redes sociales y el comportamiento participativo en Internet, consolidando una nueva estrategia de negocio que permite llegar a más personas de una manera más rápida y efectiva. Según estimaciones la economía digital colaborativa para el año 2025, moverá 235.000 millones de dólares. En América Latina esta tendencia hasta ahora está tomando forma, y al igual que a nivel global se está tratando de entender y hacer que convivan los dos enfoques, tradicional y digital, para que no riñan entre ellos, y lo que se busque es una "sana" competencia que en últimas beneficie al usuario final, por ellos los países en el mundo tienen la tarea de buscar los mejores marcos regulatorios que permitan una competencia justa entre las partes. En el caso de Colombia, este proceso se encuentra en pleno estado embrionario en materia de leyes, y en cuanto a la convivencia dado que no existe un marco legal estructurado ha permitido una serie de situaciones que han llevado a encontrar los dos esquemas económicos.

Palabras claves: economía colaborativa; consumo compartido; sociedad colaborativa

Abstract: The world economy has been undergoing major changes, which have led to the traditional economy based on hyper-consumerism being threatened and displaced by a new economic trend based on intelligent and sustainable consumption, a consumption that is concerned about not using more than what is necessary, consumption based on collaboration and the use of idle goods. This new phenomenon is derived from the economic crisis that the world was facing in the last decade, the heyday of social networks and participatory behavior on the Internet, consolidating a new business strategy that allows reaching more people in a faster and more effective According to estimates the collaborative digital economy for the year 2025, will move 235,000 million dollars. In Latin America, this trend is taking shape up to now, and just as at the global level it is trying to understand and coexist the two approaches, traditional and digital, so that they do not quarrel among themselves, and what is sought is a " healthy "competition that ultimately benefits the end user, for them the countries in the world have the task of seeking the best regulatory frameworks that allow fair competition between the parties. In the case of Colombia, this process is in full embryonic status in terms of laws, and in terms of coexistence since there is no structured legal framework has allowed a series of situations that have led to find the two economic schemes.

Key words: collaborative economy; shared consumption; collaborative society 


\section{Introducción}

A través de los años los esquemas de consumo en la economía han venido atravesando unos cambios importantes llegando a su punto máximo representado en un capitalismo hiperconsumista, cuya característica básica es la proliferación de un consumo desmesurado, que busca en lo posible que un producto sea utilizado por un solo individuo, que adicionalmente presente una alta tasa de recambio (obsolescencia programada) y que genere unas nuevas necesidades en el consumidor final (consumo emocional).

Este tipo de capitalismo, permitió de cierta manera un crecimiento económico que iba acompañado con la generación de empleo y el aumento de la producción, pero en contra parte se estaba volviendo nocivo para la mayoría y para el mismo planeta en sí, dada las nuevas tendencias de una economía inteligente y sostenible.

La Revista Time en el 2011, consideró a la Economía Colaborativa una de las 10 ideas que iban a cambiar el mundo (Walsh, 2011), y nos hacía alusión a que una decisión inteligente era no poseer sino compartir. De acuerdo a estimaciones realizadas por economistas de talla mundial y centros de estudios globales, se dice que la economía colaborativa para el año 2025, moverá 235.000 millones de dólares (Semana, 2016).

El consumo colaborativo es la base para dar inicio a la economía colaborativa, y mantiene como premisa compartir bienes antes de poseerlos, y hacer que circule lo que existe, en vez de seguir comprando cosas nuevas. Este esquema no es nuevo, ya que desde hace mucho tiempo estas acciones se realizaban en círculos cerrados, como por ejemplo, los libros que se utilizaban para la escuela, se cedían a los hermanos menores, o simplemente el hecho de compartir ropa usada o juguetes con miembros de la misma familia.

¿Pero qué ocasiona el auge de esta nueva tendencia? Entre el año 2008 y 2011 sucede una crisis económica de carácter global, lo que hizo que varios países quedaran económicamente mal, adicionalmente se estaban uniendo expresiones sociales que rechazaban el modelo económico actual ya que se consideraba inequitativo e injusto, y adicionalmente las nuevas generaciones comenzaron a tomar conciencia de la existencia de una gran cantidad de recursos, los cuales eran en gran proporción subutilizados

Sin lugar a duda existen tres detonantes que dan inicio a la economía colaborativa, tal como se encuentra concebida en la actualidad, y fueron la crisis económica, el auge de las redes sociales y los comportamientos participativos en Internet.

De acuerdo con el dictamen del Comité Económico y Social Europeo sobre "Consumo colaborativo o participativo: un modelo de sostenibilidad para el siglo XXI", esta tendencia representa una tercera ola de Internet, donde la gente se encuentra en Internet para compartir fuera de Internet, y cuya función es poner en circulación todo aquello que existe (Comité Económico y Social Europeo, 2014).

Los elementos necesarios para dar vida a la economía digital compartida son: Bienes ociosos, Tecnologías de la Información y la Comunicación, Comunidades y por último confianza y reputación. 
El término bienes ociosos hace referencia a aquellos productos que habitualmente no son muy utilizados, y que su funcionalidad se puede reactivar mediante el consumo colaborativo. Por otra parte las Tecnologías de la Información y la Comunicación (TICs) se configuran en un elemento muy importante que sirve como base o plataforma para localizar los recursos y poder conectar dinámicamente y en tiempo real la oferta y la demanda.

El término Comunidades, se refiere al conjunto de personas con intereses comunes y sistemas de pago o intercambio. Con relación a la confianza o reputación, este es un aspecto relevante entre los usuarios, ya que las referencias o comentarios serán criterios que influyen en la toma de decisiones y la actitud de otros usuarios a la hora de compartir el bien de alguna de las maneras posibles (ECODES, 2013).

\section{Economía digital compartida en Colombia}

Colombia no ha sido ajena a la llegada de esta nueva ola económica denominada economía compartida, y el problema que se está presentando es que estas nuevas formas de negocio que se han apalancado a través de las plataformas tecnológicas bajo el concepto de consumo colaborativo no tienen un marco legal vigente que permita llenar el vacío jurídico que existe, dado que las leyes colombianas están enfocadas a las formas tradicionales de negocio, y teniendo en cuenta que este movimiento es muy reciente (menos de una década) y que los avances tecnológicos son muy dinámicos es necesario crear un marco regulatorio que zanje las diferencias y permita el avance tecnológico al cual no podemos ser ajenos.

En Colombia se han presentado los proyectos de Ley 22 y 110 de 2016, que de cierta manera buscan dar bases a la economía colaborativa. El proyecto de Ley 22 de 2016, busca incluir la economía del océano azul dentro del ordenamiento legal colombiano a través de nuevas tecnologías y plataformas colaborativas, en cabeza del Gobierno Nacional. La Economía del Océano Azul, es aquella actividad económica mediante la cual se comparten e intercambian bienes y servicios a través de plataformas. Igualmente se señala que todas las personas jurídicas que deseen prestar algún servicio o intercambiar algún tipo de bien a través de plataformas, deberán contar con un registro mercantil conforme lo determinan las leyes preexistentes (Garcia Zuccaerdi, Chamorro, Tamayo, \& Osorio, 2016).

El proyecto de Ley 110 de 2016, busca regular el trabajo autónomo económicamente dependiente en Colombia, con dicha iniciativa se busca proteger el trabajo desarrollado por los trabajadores autónomos económicamente dependientes (TAED), permitiendo expresamente que se asocien frente a cada plataforma de economía colaborativa o a modo de gremio por servicios. Son trabajadores autónomo económicamente dependiente aquellas personas naturales que realicen de forma habitual, personal, directa y sin subordinación en el ámbito de dirección y organización de una plataforma de economía colaborativa, una actividad económica o profesional a título lucrativo y que represente para el trabajador por lo menos un ingreso mensual de un salario mínimo legal mensual vigente. Los TAED son una parte integral del modelo económico de las plataformas móviles de economía colaborativa (Lara Restrepo, 2016).

\section{Diferencias con las demás figuras del ordenamiento jurídico colombiano}




\begin{tabular}{|c|c|c|}
\hline El trabajador & El contratista independiente & El Trabajador autónomo \\
\hline Código Sustantivo del Trabajo & Código Civil & económicamente dependiente \\
\hline $\begin{array}{l}\text { Subordinación jurídica Laboral y } \\
\text { dependencia económica del } \\
\text { empleador }\end{array}$ & Independencia jurídica & $\begin{array}{l}\text { Independencia jurídica y dependencia } \\
\text { económica frente a la plataforma de } \\
\text { economía colaborativa }\end{array}$ \\
\hline Prestaciones sociales y & N/A & N/A \\
\hline vacaciones & & \\
\hline Horarios fijos & N/A & $\begin{array}{l}\text { No hay horarios fijos ni control sobre los } \\
\text { mismos por parte de la plataforma de } \\
\text { economía colaborativa }\end{array}$ \\
\hline $\begin{array}{l}\text { Ius variandi (Poder patronal de } \\
\text { variación) }\end{array}$ & N/A & N/A \\
\hline Pensiones, Salud y ARL a & Pensiones, Salud y ARL a & Pensiones, Salud y ARL por mitades \\
\hline $\begin{array}{l}\text { prorrata entre empleador y } \\
\text { Trabajador }\end{array}$ & independiente & \\
\hline Salario & Honorarios pactados fijos & $\begin{array}{l}\text { Control del porcentaje y tarifa del } \\
\text { servicio por parte de la plataforma de } \\
\text { economía colaborativa }\end{array}$ \\
\hline $\begin{array}{l}\text { Indemnización por despido sin } \\
\text { justa causa }\end{array}$ & N/A & N/A \\
\hline $\begin{array}{l}\text { Hace parte integral -como pieza } \\
\text { fundamental-del objeto misional } \\
\text { del empleador }\end{array}$ & N/A & $\begin{array}{l}\text { Hace parte integral -como pieza } \\
\text { fundamental- del objeto misional de la } \\
\text { plataforma de economía colaborativa }\end{array}$ \\
\hline
\end{tabular}

Es importante tener en cuenta que a la fecha ninguno de estos dos proyectos de ley se han convertido en leyes de la República, y aún se encuentran en trámites legislativos, pero esto solo es un pequeño paso, dado que faltan muchos aspectos por cubrir dada la diversidad de tipos de servicios que se están ofreciendo, y dado la naturaleza que tiene cada uno de ellos será necesario regularnos casi que de forma individual, tal como por ejemplo, lo que se ha presentado en la prestación de servicios de transporte o alojamiento por medio de plataformas como Uber o AirBnb, en donde no se ha logrado un acuerdo entre los actores que se encuentran involucrados, y en últimas ha generado una serie de controversias que no han permitido una armónica convivencia.

Estos nuevos modelos de negocio están cambiando el modelo de empleo tradicional y se está convirtiendo, por lo menos para el caso colombiano, en el sustento de un cúmulo de personas, que dada la dificultad para ingresar en el mercado laboral formal, encuentran en este modelo una forma de obtener ingresos para de esta manera llevar el sustento a sus hogares.

En un estudio realizado por el IE Business School, en el cual realizaron una encuesta entre agosto y noviembre de 2015 contando con la participación de 107 empresas, buscó caracterizar la Economía Colaborativa en América Latina (Multilateral Investment Fund \& IE Business School, 2016), obteniendo algunos resultados interesantes como por ejemplo de acuerdo al lugar de fundación de la empresa, Brasil lidera con un 32\%, seguido por Argentina y México con un $13 \%$ cada uno, mientras que Colombia tenía un 9\%.

Con relación al tipo de actividad que facilita la iniciativa realizada, en un $27 \%$ era de Alquiler (Renting). En lo Concerniente al número de personas que participan en la iniciativa, un $35 \%$ manifestó que de una a cinco personas. Entre los fines principales que movieron la creación 
de las iniciativas estuvieron: crear nuevas formas de economía, mejorar la calidad de vida de las personas, mejorar la economía local, utilizar mejor recursos infrautilizados.

En los últimos dos años Colombia ha avanzado en la incorporación de la economía colaborativa, para el año 2017, de acuerdo a lo dicho por el Ministro de Tecnologías de la Información y las Comunicaciones, Colombia se está convirtiendo en un país líder de la región en la generación y presencia de iniciativas de economía colaborativa, soportada en plataformas tecnológicas (El Tiempo, 2017).

En Colombia ya se cuenta con un Hub y Laboratorio de Economía Colaborativa llamado Sharecollab, que hace parte de Ouishare, quien es la comunidad global y organización líder en la Economía Colaborativa, quien de acuerdo a su fundador y CEO Gustavo Palacios ya se han reunido 200 proyectos colaborativos en Colombia.

Hoy por ejemplo el "problema" es Uber con su modelo de negocio, y es una amenaza para el gremio de conductores de taxis, pero no estamos muy lejos de que ahora los vehículos sean autónomos, luego el enemigo será otro.

Lo importante ahora es establecer normas regulatorias que permitan convivir los modelos tradicionales con los digitales, que permitan ofrecer equidad, y una competencia más justa, prevaleciendo en primer lugar los derechos que tenga el consumidor, y de esta manera evitar confrontaciones entre todos los actores involucrados, y conjuntamente se deben establecer condiciones ecuánimes que permitan una sana competencia.

\section{Conclusiones}

Es necesario comenzar a evolucionar en nuestro marco legal, dado que debemos ir a la par con los avances tecnológicos, no podemos ir contra ellos, debemos comenzar a regular desde lo actual, pero complementando y permitiendo que esta nueva tendencia de economía colaborativa tenga un mejor desarrollo, ya que en últimas los mayores beneficiados serán los usuarios que tendrán una oferta más amplia de servicios, que permitirán una libre competencia, y ocasionará que los modelos de negocios tradicionales se re-piensen o re-inventen con el objetivo de ser más competitivos.

No podemos pretender cortar las alas de los avances tecnológicos, dado que el crecimiento de los mismos se está dando de forma exponencial, y cada día se presentaran nuevos retos, a los cuales debemos tener la capacidad de afrontarlos con unos marcos regulatorios flexibles y en algunos casos podrían ser personalizados.

Para el caso de Colombia, la economía colaborativa ha avanzado a pasos agigantados en cuanto al número de aplicaciones tecnológicas, mientras que en materia regulatoria los avances han sido bastante lentos, y no han obedecido a la dinámica del entorno, lo cual es contraproducente al momento de impulsar la economía digital en el país, y sobre todo si se persiste en pensar y legislar basados en modelos tradicionales de negocios. 


\section{Bibliografía}

Comité Económico y Social Europeo. (Enero de 2014). http://eur-lex.europa.eu. Obtenido de http://eur-lex.europa.eu/legal-content/ES/TXT/?uri=CELEX\%3A52013IE2788

ECODES. (Septiembre de 2013). www.ecode.org. Obtenido de http://ecodes.org/component/option,com_phocadownload/Itemid,2/download,230/id,14/vie w,category/

El Tiempo. (23 de Enero de 2017). Plataformas que se imponen en el mundo del consumo colaborativo. El Tiempo. Obtenido de http://www.eltiempo.com/tecnosfera/novedadestecnologia/plataformas-de-economia-colaborativa-38468

Garcia Zuccaerdi, A., Chamorro, J., Tamayo, J., \& Osorio, H. (Julio de 2016). Congreso de la República de Colombia. Obtenido de http://190.26.211.102/proyectos/index.php/textosradicados-senado/pl-2016-2017/675-proyecto-de-ley-022-de-2016

Lara Restrepo, R. (Agosto de 2016). Congreso de la República de Colombia. Obtenido de http://190.26.211.102/proyectos/index.php/textos-radicados-senado/pl-2016-2017/759proyecto-de-ley-110-de-2016

Multilateral Investment Fund, \& IE Business School. (2016). Economía Colaborativa en América Latina. IE Business School. Obtenido de informeeconomiacolaborativalatam.ie.edu/informe-economia-colaborativa.pdf

Semana. (19 de Marzo de 2016). Semana. Obtenido de http://www.semana.com/economia/articulo/uber-airbnb-y-bitcoin-economia-colaborativaamenaza-a-la-tradicional/465955

Walsh, B. (17 de Marzo de 2011). TIME. Obtenido de http://content.time.com/time/specials/packages/article/0,28804,2059521_2059717_205971 0,00.html 